\title{
Prospect of Immunotherapy for Glioblastoma: Tumor Vaccine, Immune Checkpoint Inhibitors and Combination Therapy
}

\author{
Eiichi ISHIKAWA, ${ }^{1}$ Tetsuya YAmamoto, ${ }^{1}$ and Akira MATSUMURA ${ }^{1}$ \\ ${ }^{1}$ Department of Neurosurgery, Faculty of Medicine, University of Tsukuba, \\ Tsukuba, Ibaraki, Japan
}

\begin{abstract}
To date, clinical trials of various vaccine therapies using autologous tumor antigens or tumor-associated/ specific antigen peptide with adjuvants have been performed to treat patients with high-grade gliomas (HGG). Furthermore, immune checkpoint pathway-targeted therapies including anti- programmed cell death 1 (PD-1) antibody have been remarkably effective in other neoplasms, and various clinical trials with anti-PD-1 antibody in patients with HGG have started to date. It is possible that up-regulation of immune checkpoint molecules in tumor tissues after vaccine therapy may be one of the mechanisms of vaccine failure. Multiple preclinical studies indicate that combination therapy with vaccination and immune checkpoint blockade is effective for the treatment of malignant tumors including HGG. Thus, immunotherapy, especially combination therapy with vaccine and immune checkpoint inhibitors, may be a promising strategy for treatment of patients with HGG.
\end{abstract}

Key words: brain tumor immunotherapy, glioblastoma, autologous formalin-fixed tumor vaccine, PD-1, immune checkpoint inhibitor

\section{Introduction}

To improve the dismal prognosis of patients with high-grade gliomas (HGG) including glioblastoma (GBM), chemotherapy including temozolomide (TMZ) combined with radiation therapy (RT) has been a standard treatment. ${ }^{1,2)}$ On the other hand, a growing interest in new treatments including immunotherapy such as vaccine-based therapy has emerged. ${ }^{3-15)}$ The main concept of these immunotherapies is enhancement of anti-tumor-specific immune reactions and activation of tumor-specific killer cells, the so-called cytotoxic T-lymphocytes (CTLs) (Fig. 1). Here, we first describe the adoptive immunotherapies used for HGG in our institution. Second, we describe clinical trials of autologous formalin-fixed tumor vaccine (AFTV) therapies, which may be easier than adoptive immunotherapies in terms of their preparation and may have some benefits in longstanding effectiveness and cost. We also discuss the combination of vaccine therapy with immune checkpoint pathway-targeted therapies.

Received December 27, 2016; Accepted February 19, 2017
Adoptive immunotherapy for HGG in our institution and the paradigm shift to vaccine therapy

Several authors have reported the safety and efficacy of lymphokine-activated killer (LAK) cell therapy in patients with HGG. ${ }^{16-19)}$ Because the natural killer (NK) cell component among LAK cells has strong cytotoxic activity against malignant tumor cells, we previously performed a clinical trial of NK cell therapy using a novel method of NK cell expansion for patients with HGG. ${ }^{20)}$ Among several immune cell types associated with acquired immunity, immune cells, such as autologous tumor-specific T-lymphocytes (ATTLs) including CTLs, have also been considered to play a crucial role in tumor rejection in vivo. ${ }^{21)}$ However, these cell therapies have some issues, including a short duration of treatment response, complexity of ex vivo cell expansion, and expense. ${ }^{17,20,21)}$ Such issues may be the reason for a recent paradigm shift from cell therapy to vaccine therapy as a treatment tool for HGG.

A strong demand for adoptive immunotherapy still exists today because a relatively higher tumor response can be expected to immunotherapy than to vaccine therapy, ${ }^{7,20,21)}$ despite the major disadvantages described above. Moreover, activation of new legislation 


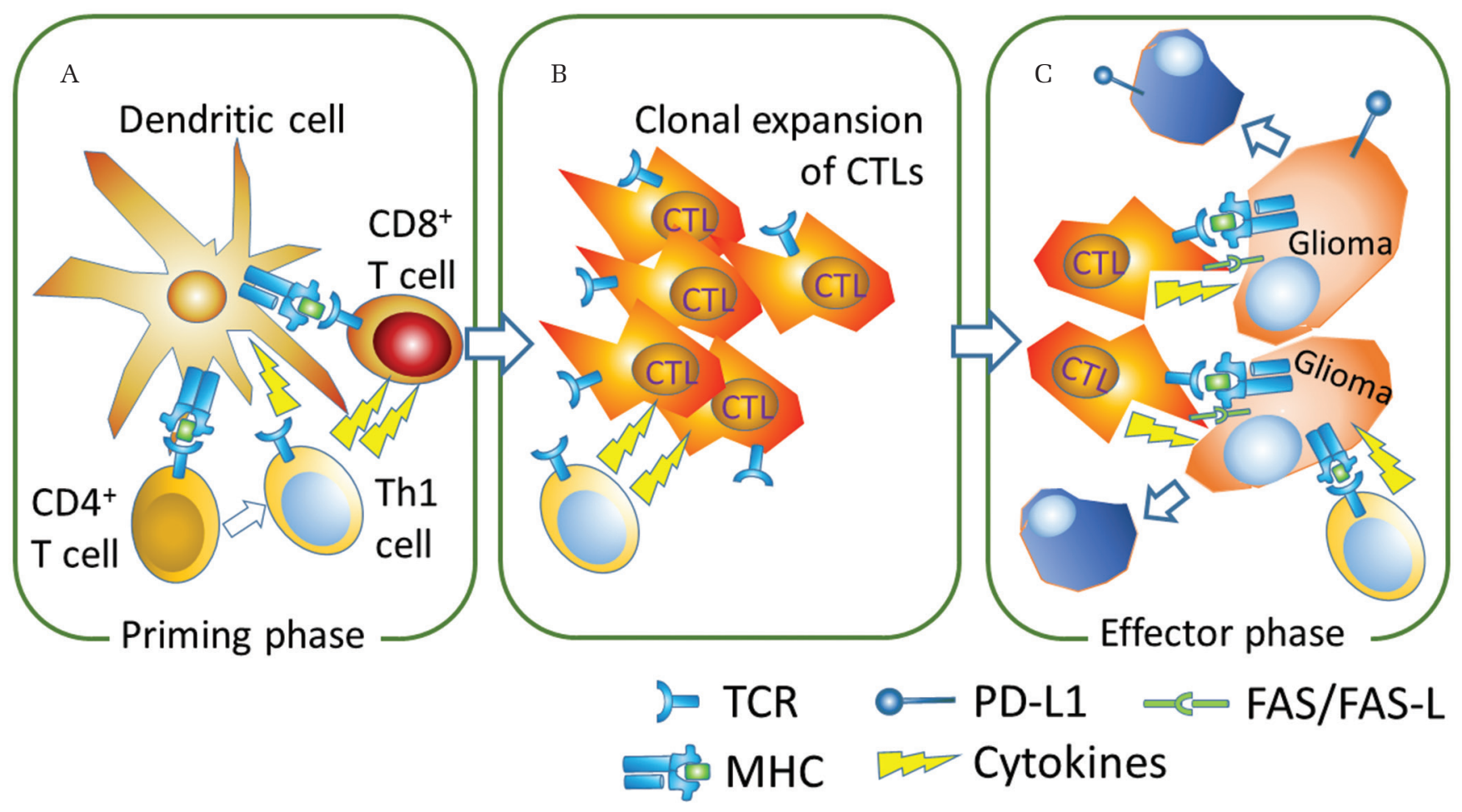

Fig. 1 Induction of activated CD8+ cytotoxic $T$ lymphocytes (CTLs) in vaccine therapy. A. In the priming phase, CD8+ T cells are activated by dendritic cells (DCs) stimulated with tumor vaccination. Binding of the major histocompatibility complex (MHC) with tumor antigen and the $\mathrm{T}$ cell receptor (TCR) and interaction of costimulating molecules are required for this activation. Type 1 helper $T$ cells (Th1) also work as an activator for CD8 $T$ cells. B. Clonal expansion of activated CTLs. C. In the effector phase, the activated CTLs mediate tumor-specific MHC class 1-restricted lysis of glioma cells, production of cytokines (e.g., Interferon (IFN)- $\gamma$ and tumor necrosis factor- $\alpha$ ) and upregulation of Fas ligand.

and facilitation of the entry of new companies into the research market for immunotherapeutic reagents have prompted the practical promotion of regenerative medicine including adoptive cell transfer of these immune cells as well as the recently described induced pluripotent stem cells. Combination of adoptive immunotherapy with chemotherapy or with vaccine therapy, has been attempted in many studies. ${ }^{17,22)}$ Recently, as a new strategy, adoptive immunotherapy after chemotherapy-induced lymphodepletion has been used to treat other neoplasms. ${ }^{22)}$ Lymphodepletion seemed to enhance the antitumor effects of transferred $\mathrm{T}$ cells in vivo by several mechanisms including the elimination of suppressive $\mathrm{CD} 4^{+} \mathrm{CD} 25^{+}$T-regulatory lymphocytes (Tregs), the elimination of cellular "sinks" for homeostatic cytokines such as IL-7 and IL-15, and the engagement of toll-like receptors on antigen-presenting cells after damage to the integrity of the gut epithelial lining. ${ }^{22)}$ Modified LAK/CTL therapy using a chimeric antigen receptor (CAR) has received much attention as the next generation of adoptive immunotherapy. A clinical trial of this CAR therapy has already begun for treatment of other carcinomas, ${ }^{23)}$ and future clinical applications are also expected for HGG. ${ }^{24,25)}$

\section{Tumor vaccine therapy for HGG}

To date, clinical trials of various vaccine therapies using autologous tumor antigens or tumorassociated/specific antigen peptide with adjuvants have been performed to treat patients with HGG. ${ }^{3-15)}$ The types of peptides used include an epidermal growth factor receptor variant III (EGFRvIII) peptide, an Wilms tumor 1 (WT1) peptide and personalized tumor-associated/specific antigen peptides. Peptide-based vaccine therapies appear promising as an approach to successfully induce an antitumor immune response and prolong survival in patients with glioma, without major side effects. ${ }^{13)}$ In a phase II study of an autologous heat-shock protein peptide complex-96 (HSPPC-96) vaccine for surgically resectable recurrent GBM patients, the median overall survival (OS) was 9.9 months with no treatment-related deaths. ${ }^{3)}$ A double-blind, phase III trial of rindopepimut consists of EGFRvIII peptide conjugated to keyhole limpet hemocyanin in 
patients with newly diagnosed, EGFRvIII-expressing GBM did not show significant survival benefit for patients with minimal residual disease despite consistent rindopepimut-induced humoral response. A trend for improved long-term survival was observed in patients with bulkier disease, but this was not supported by further analyses within this subgroup. ${ }^{14)}$ Clinical research with dendritic cell (DC) therapy is also being performed. ${ }^{5,26)}$ DC therapy for prostate cancer patients (PROVENGE) has been approved by the U.S. Food and Drug Administration (FDA). ${ }^{27)}$ Such DC therapy is theoretically very promising if the complexity of DC culture can be overcome.

We carried out a pilot clinical trial. ${ }^{7)}$ using a novel immunotherapy, AFTV, that consisted of autologous formalin-fixed tumor fragments and adjuvants developed by T Ohno, RIKEN, Japan. ${ }^{28)}$ In this pilot study, of 12 GBM patients, including 8 recurrent disease and 4 primary disease with visible tumor mass, the only side effects of the treatment were local erythema, induration, and low-grade fever. ${ }^{7)}$ Low p53 and high major histocompatibility complex (MHC) class-I expression by the tumor were related with the efficacy of this therapy in this study. In our first prospective multicenter trial for preliminary evaluation of the efficacy of AFTV concomitant with fractionated radiotherapy (FRT) in patients with newly diagnosed GBM (study identification number: C000000002) (Fig. 2A). this treatment resulted in a median OS duration of 19.8 months and an actuarial 2-year survival rate of $40 \% .^{9)}$

During this clinical study, TMZ combined with FRT became a standard treatment for GBM. Although TMZ frequently induces a decrease in lymphocyte count, ${ }^{29}$ TMZ-induced lymphopenia enhances tumor-specific immune responses. ${ }^{30}$ Animal studies have shown that TMZ has the potential to enhance antitumor immunity. ${ }^{31,32)}$ Preclinical studies have shown that vaccination with DCs followed by chemotherapy can significantly increase survival in patients with malignant gliomas. ${ }^{33}$ These results together with practicable results in a pilot study using AFTV and $\mathrm{TMZ}^{34)}$ prompted us to proceed with a further phase I/IIa multicenter trial (UMIN000001426) of AFTV and maintenance TMZ after FRT concomitant with TMZ for management of newly diagnosed GBM. ${ }^{6)}$ (Fig. 2B). The primary endpoint in this study was OS, and the secondary endpoint was PFS. Eligibility

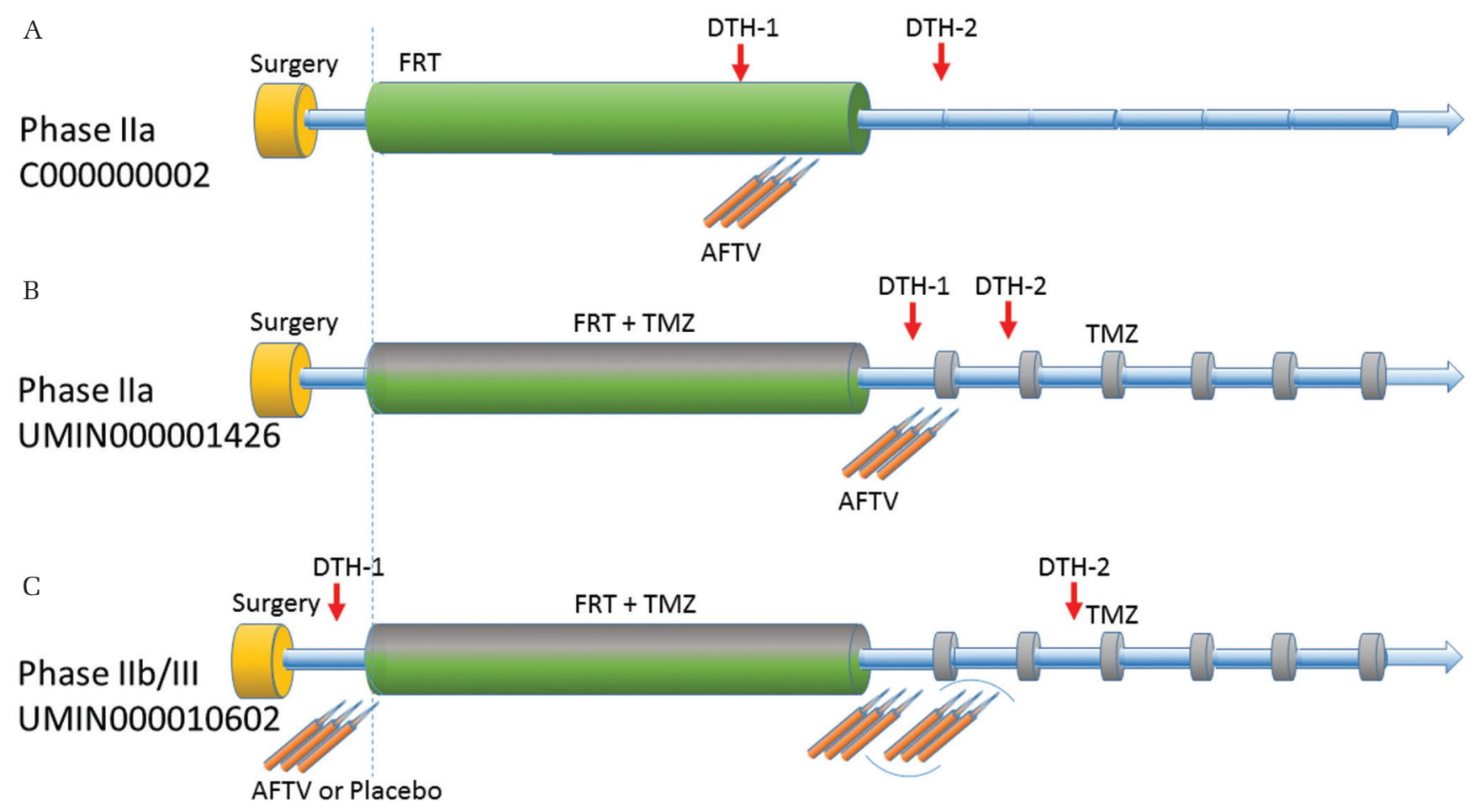

Fig. 2 Three prospective multicenter trials for evaluation of the efficacy of autologous formalin-fixed tumor vaccine (AFTV) for patients with initially diagnosed glioblastoma (GBM). A. C000000002 trial is a single arm phase IIa study using AFTV concomitant with fractionated radiotherapy (FRT) without chemotherapy ${ }^{9}$. B. UMIN000001426 trial is a single arm phase IIa study using FRT concomitant with temozolomide (TMZ) followed by AFTV and maintenance $\mathrm{TMZ}^{6)}$. C. UMIN000010602 trial is an on-going double-blinded randomized phase IIb/III study using 6 to 9-time injection of AFTV (or placebo) and FRT concomitant with TMZ. 
criteria included patients who were 16-75 years of age, newly diagnosed GBM with histopathological confirmation of diagnosis of the disease with typical neurological symptoms, maximum possible resection of the tumor, and a Karnofsky performance status scale (KPS) score of $\geq 60 \%$ before initiation of FRT. All enrolled patients received the standard Stupp regimen ${ }^{2)}$ with a modified TMZ maintenance cycle. Briefly, FRT was started within 2-3 weeks after resection of the neoplasm and included 60 Gy of focal irradiation, with daily concurrent TMZ throughout the course of radiotherapy. At the end of the induction period, after a 4-week interval, the patients received TMZ maintenance chemotherapy for 5 days in each 28-day cycle. The AFTV treatment consisted of three courses of vaccination performed at 1-week intervals. The first course corresponded to the first day of TMZ maintenance chemotherapy. Of the 24 enrolled patients, 8 were recursive partitioning analysis (RPA) class III, 10 were class IV, and six were class V. Although this treatment did not have a great impact on the median PFS of the patients (8.2 months) the 2-year progression-free rate was $33 \% .{ }^{14)}$ PFS showed a statistically significant association with patient age, KPS score, RPA class, extent of tumor removal, and delayed-type hypersensitivity response after vaccination (DTH-2) in the data of the 24 patients enrolled in the study. The duration of PFS in patients with a DTH-2 response of $10 \mathrm{~mm}$ or larger was significantly longer than in patients with a smaller DTH-2 response. The median OS of the 24 patients in this study was 22.2 months. The actuarial 2- and 3-year survival rates were $47 \%$ and $38 \%$, respectively, at the time of submission of the manuscript of this study. ${ }^{6}$ ) Moreover, comparison of OS in subgroups divided by lymphopenia grade that was probably due to TMZ, patients with moderate lymphopenia (CTCAE grade 3) showed better survival than those with less severe lymphopenia, indicating that TMZ-induced lymphopenia may enhance tumor-specific immune responses in this study. ${ }^{6}$ Also in the long-term follow-up data of the 24 patients, the median OS was 22.2 months. The actuarial 2- and 3-year survival
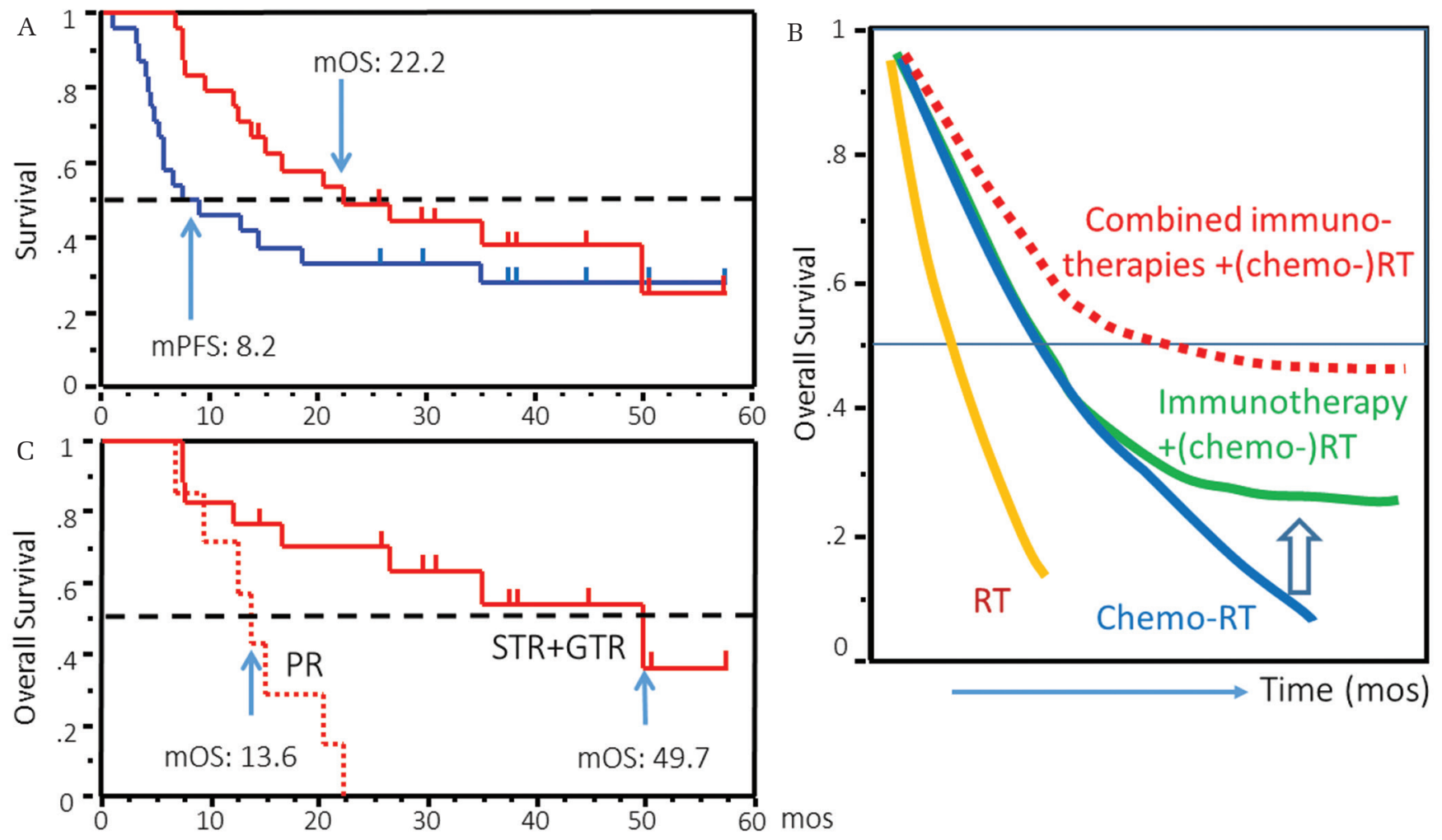

Fig. 3 Outcome of GBM patient treated with AFTV. A. Overall survival (OS) and progression-free survival (PFS) curves using long-term follow-up data of patients with initially diagnosed GBM enrolled in UMIN000001426 trial $^{35)}$. B. Expected image of OS curves of GBM patients treated with radiotherapy (RT) (yellow line), chemotherapy plus RT (blue line), combination of immunotherapy with (chemo-) RT (green line), and combination of several different types of immunotherapies (e.g., vaccine and an immune checkpoint inhibitor) with (chemo-) RT (red dotted line). C. OS of patients with sub-totally or gross-totally resected (STR+GTR, 98\% or more of extent of resection ratio) and partially resected (PR) GBM in UMIN000001426 trial $^{35)}$. 
rates were $49 \%$ and $38 \%$, respectively. ${ }^{35)}$ (Fig. 3A). This high percentage of long term survivors may be a significant feature of this study (Fig. 3B). OS in patients that underwent subtotal or total tumor removal (98\% or more removal ratio) was better than that in patients who underwent partial removal ${ }^{35)}$ (Fig. 3C). Interestingly, DTH-2 was associated with the extent of tumor removal in 45 patients treated with AFTV (C000000002 and 000001426 studies). This result indicates that maximal surgical removal of the tumor may be imperative for vaccine therapy to improve tumor-specific immune reactions.

These studies also indicate that AFTV using autologous formalin-fixed tumor tissue fragments is as safe as peptide vaccines ${ }^{3-15)}$ and that the use of the fragments as tumor antigens may not be inferior to single peptide vaccines or a combination of various peptides in GBM. Interestingly, in a patient with advanced biliary tract cancer who was receiving a peptide vaccine after the standard chemotherapy plus AFTV, strong immune and clinical responses were observed over the long term. ${ }^{36)}$ Thus, AFTV before (or after) peptide vaccine(s) may also boost tumor-specific immune reactions by peptide vaccine(s) in GBM patients. Since March 2013, we have started a double-blinded randomized phase IIb/III trial of AFTV with TMZ for newly diagnosed GBM to evaluate the efficacy of AFTV for prevention of recurrence and/or cure of residual tumor (UMIN000010602) (Fig. 2C). The primary outcome is OS and key secondary outcomes are cause-specific survival, PFS, tumor-regression rate, disease-control rate, quality of life and neurological improvement.

\section{Immune checkpoint inhibitors for HGG and combination immunotherapy}

Immune checkpoint inhibitors are attracting attention in HGG, as they are in other cancers. Among immune checkpoint pathways, programmed cell death 1(PD-1) is one of the most promising key molecules and is essential not only for maintaining self-tolerance under physiological condition, but also for generating the inhibitory immune microenvironment for the tumor. ${ }^{37,38)}$ In most cases of HGG, PD ligand-1 (PD-L1, also known as B7-H1) is expressed in glioma cells. PD-L1 not only reduces interferon (IFN)- $\gamma$ production by activated $\mathrm{T}$ cells, but also drives the differentiation of naïve CD4 T cells into Tregs. ${ }^{38)}$ High PD-L1/ PD-1 expression is thought to be a prognostic marker in some types of cancers. ${ }^{39,40)}$ Similarly, in GBM, a study that analyzed $92 \mathrm{GBM}$ patient tissues showed that high expression of PD-L1 on GBM cells was a poor prognostic marker. ${ }^{41)}$ In contrast, another study that analyzed tissue from 117 initially diagnosed and 18 recurrent GBM patients showed no correlation between high PD-L1 expression and prognosis. ${ }^{42)}$ In our study, which investigated PD-1 expression in GBM specimen pairs at the initial stage and recurrent stage after RT plus TMZ therapy at our institute, an increase in the number of PD-1-positive cells in the latter specimens was associated with worse outcome after recurrence. ${ }^{43)}$

The clinical use of anti-PD-1 antibody (pembrolizumab (lambrolizumab) or nivolumab) is remarkably effective in other neoplasms. ${ }^{44-46)}$ A phase IIb, randomized, open-label study to evaluate the efficacy and safety of nivolumab alone or together with anticytotoxic T-lymphocyte antigen 4 (CTLA-4) antibody (ipilimumab) versus bevacizumab in patients with recurrent GBM has begun. ${ }^{47}$ To date, various clinical trials of checkpoint inhibitors in patients with initiallydiagnosed HGG have also been initiated. ${ }^{38)}$ Examples of ongoing studies are a randomized phase II single blinded study (CheckMate 548) of TMZ plus radiation therapy combined with nivolumab or placebo in newly diagnosed adult subjects with O6-methylguanine-DNA methyltransferase (MGMT)-methylated GBM, and a randomized phase III open label study (CheckMate 498) of nivolumab versus TMZ, each in combination with radiation therapy in newly diagnosed adult subjects with unmethylated MGMT GBM. ${ }^{48,49)}$ Excellent clinical results of immunotherapies for HGG using these antibodies are expected.

Even if cytotoxic CD8-positive tumor infiltrating lymphocytes (TILs) are induced after tumor vaccination, in practice, glioma cells recur in most cases of GBM. One of the mechanisms of this immunotherapy failure is tumor escape, due to decreased antigen and MHC class I expression, and an increased number of suppressor cells around the tumor cells. ${ }^{50)}$ To date, chemotherapy and radiotherapy have been thought to modulate the tumor microenvironment to enhance immunotherapy for various neoplasms ${ }^{51)}$ through a reduction in tumor volume. Similarly, in a murine glioma model, the combination of radiotherapy and vaccination results in long-term survival, ${ }^{52)}$ and crosspriming by TMZ enhances the antitumor immunity of dendritic cell vaccination. ${ }^{53)}$ However, the best practical combination of tumor vaccine therapies with surgery, chemotherapy, and radiotherapy for HGG is still unclear and is likely to be an active area of research in the future. Active angiogenesis may be a reason why tumors are refractory against chemoradiotherapy in HGG. Some animal studies using glioma cells and other neoplasms have demonstrated that anti-angiogenic therapy enhances immunotherapy, ${ }^{54-57)}$ due to re-engineering of the tumor-immune microenvironment via decreasing immunosuppressive cells, immunosuppressive cytokines and inhibitory molecules including PD-1 on T cells. ${ }^{57}$ 
These studies indicate that combined anti-angiogenesis therapy with immunotherapy may be a reasonable therapeutic strategy.

Up-regulation of immune checkpoint molecules in tumor tissues after immunotherapy may also be a mechanism of this vaccine failure especially in the recurrent phase (Fig. 4). IFN- $\alpha$ and IFN- $\gamma$ secreted by activated $\mathrm{T}$ cells mediate PD-1 expression on TILs and PD-L1 expression on tumor cells, respectively. ${ }^{58-60)}$ Similarly, in our study of GBM specimen pairs at the initial phase and the recurrent phase after post-surgical treatment with and without AFTV, PD-1 expression scores on TILs were significantly increased, and a trend towards increasing PD-L1 expression was detected in tumor cells in the latter specimens of the vaccine group compared with the initial resection specimens. ${ }^{43)}$ These results indicate that anti-PD-1 antibody therapy may be effective in preventing tumor recurrence after initial surgery. Some preclinical investigations support this speculation.
Combination therapy with vaccination and antiPD-1 antibody in murine cancer models enhanced TIL antigen reactivity and prolonged survival of tumor-bearing mice. ${ }^{61,62)}$ Combination therapy with vaccination and dual or triple immune checkpoint blockade inhibited tumor growth of cancers more effectively than monotherapy or combination therapy with single immune checkpoint blockade. ${ }^{63,64)}$ Clinical effects of CTLA-4 antibody in previously vaccinated cancer patients have been shown. ${ }^{65}$ Combination therapy with AFTV, anti-PD-1 antibody and radiation was effective for the treatment of chemo-refractory liver-metastasized uterine cervical small cell carcinoma. ${ }^{66)}$ Although no clinical case of HGG treated with immune checkpoint inhibitors combined with vaccine has been reported clinically, PD-1 antibody combined with radiotherapy produce long-term survival in a murine glioma model. ${ }^{67,68)}$ Combination therapy of a double immune checkpoint blockade also prolonged survival of mice

A

B

C
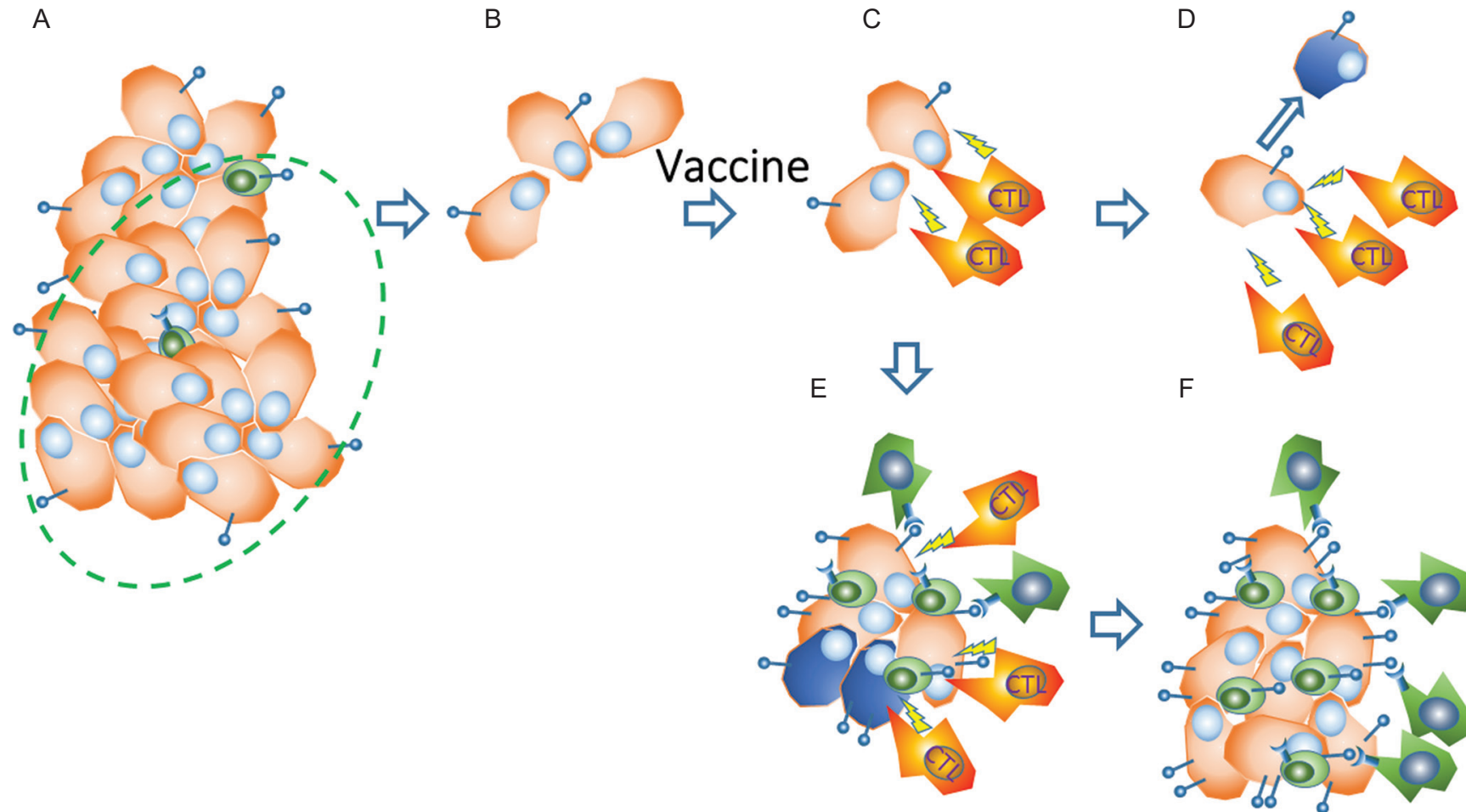

F

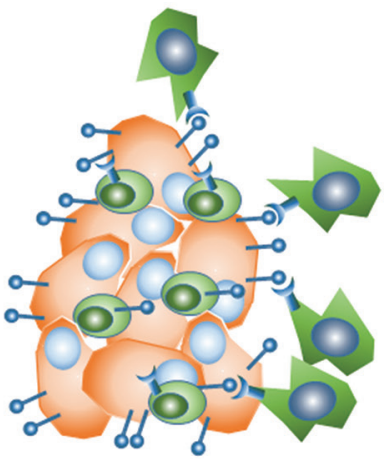

Fig. 4 Up-regulation of immune checkpoint molecules in tumor tissues after immunotherapy in vaccine failure. A. A glioma tissue consisting of massive high-grade glioma (HGG) cells (brown) and tumor infiltrating lymphocytes (TILs) that mainly consist of suppressor cells (green). B. The HGG tissue is debulked with surgical removal (dotted circle in Fig. A) followed by chemo-radiotherapy. C. Activated CTLs induced by tumor vaccination mediate tumor-specific MHC class 1-restricted lysis and production of cytokines (yellow arrows of lightning) in response to HGG cells. D. A dramatic decrease or disappearance of the glioma cells occurs in long-term recurrence-free cases after the tumor vaccine. E. In other cases, some of the CTLs are inactivated (or exhausted) even if surviving glioma cells exist, due to upregulated expression of immune checkpoint molecules including programmed death (PD)-1 on immune cells and PD-1 ligands on glioma cells through the IFNs produced by the activated CTLs themselves. F. Vaccine-failure state of tissue with recurrent glioma cells, suppressor cells and inactivated CTLs. 
with brain tumors. ${ }^{69)}$ Clinically, we need to pay attention to serious immune-related adverse events (AEs) after treatment with each immune checkpoint inhibitor, ${ }^{70,71)}$ and increasing of AEs in combination of immune checkpoint inhibitors. ${ }^{72)}$ For instance, the incidence of treatment-related AEs of grade 3 or 4 was higher in the nivolumab-plus-ipilimumab group $(55.0 \%)$ than in the nivolumab group (16.3\%) or the ipilimumab group $(27.3 \%)$ in a randomized, double-blind, multicenter, phase 3 trial (CheckMate 067) of previously untreated metastatic melanoma. ${ }^{72)}$

We therefore expect that combination of tumor vaccine with other treatments including anti-PD-1 antibody will be an effective strategy for glioma immunotherapy. As described above, immunotherapies including tumor vaccine therapy for HGG are steadily improving. We hope that immunotherapy will be incorporated as part of the standard treatment for HGG in the future.

\section{Acknowledgment}

The authors deeply thank Professor Koji Tsuboi, Proton Medical Research Center, University of Tsukuba, Professor Yoshihiro Muragaki, Faculty of Advanced Techno-Surgery, Institute of Advanced Biomedical Engineering and Science, Graduate School of Medicine, Tokyo Women's Medical University, and Tadao Ohno, Cell-Medicine, Inc. for their kind supervision regarding the author's immunotherapeutic studies.

\section{Conflicts of Interest Disclosure}

The authors have declared that no conflict of interest exists. This study was supported by projects for Grant-in-Aid for Scientific Research of Japan and promoting practical applications of advanced medical technologies in Tsukuba University Hospital.

\section{References}

1) Committee of Brain Tumor Registry of Japan. Report of brain tumor registry of Japan (2001-2004) 13th Edition. Neurol Med Chir (Tokyo) 54 (Suppl): 9-102, 2014

2) Stupp R, Hegi ME, Mason WP, et al.; European Organisation for Research and Treatment of Cancer Brain Tumour and Radiation Oncology Groups; National Cancer Institute of Canada Clinical Trials Group: Effects of radiotherapy with concomitant and adjuvant temozolomide versus radiotherapy alone on survival in glioblastoma in a randomised phase III study: 5-year analysis of the EORTC-NCIC trial. Lancet Oncol 10: 459-466, 2009
3) Bloch O, Crane CA, Fuks Y, et al.: Heat-shock protein peptide complex-96 vaccination for recurrent glioblastoma: a phase II, single-arm trial. Neuro-oncology 16: 274-279, 2014

4) Del Vecchio CA, Li G, Wong AJ: Targeting EGF receptor variant III. tumor-specific peptide vaccination for malignant gliomas. Expert Rev Vaccines 11: 133-144, 2012

5) Hashimoto N. Cancer Immunotherapy for Gliomas: Overview and Future Directions. Neurol Med Chir (Tokyo) 56: 355-360, 2016

6) Ishikawa E, Muragaki Y, Yamamoto T, et al.: Phase I/IIa trial of fractionated radiotherapy, temozolomide, and autologous formalin-fixed tumor vaccine for newly diagnosed glioblastoma. J Neurosurg 121: 543-553, 2014

7) Ishikawa E, Tsuboi K, Yamamoto T, et al.: Clinical trial of autologous formalin-fixed tumor vaccine for glioblastoma multiforme patients. Cancer Sci 98: 1226-1233, 2007

8) Izumoto $\mathrm{S}$, Tsuboi $\mathrm{A}$, Oka $\mathrm{Y}$, et al.: Phase II clinical trial of Wilms tumor 1 peptide vaccination for patients with recurrent glioblastoma multiforme. J Neurosurg 108: 963-971, 2008

9) Muragaki Y, Maruyama T, Iseki H, et al.: Phase I/IIa trial of autologous formalin-fixed tumor vaccine concomitant with fractionated radiotherapy for newly diagnosed glioblastoma. Clinical article J Neurosurg 115: 248-255, 2011

10) Pollack IF, Jakacki RI, Butterfield LH, et al.: Antigen-specific immune responses and clinical outcome after vaccination with glioma-associated antigen peptides and polyinosinic-polycytidylic acid stabilized by lysine and carboxymethylcellulose in children with newly diagnosed malignant brainstem and nonbrainstem gliomas. J Clin Oncol 32: 2050-2058, 2014

11) Reardon DA, Wucherpfennig KW, Freeman G, et al.: An update on vaccine therapy and other immunotherapeutic approaches for glioblastoma. Expert Rev Vaccines 12: 597-615, 2013

12) Steiner HH, Bonsanto MM, Beckhove P, et al.: Antitumor vaccination of patients with glioblastoma multiforme: a pilot study to assess feasibility, safety, and clinical benefit. J Clin Oncol 22: 4272-4281, 2004

13) Terasaki M, Shibui S, Narita $Y$, et al.: Phase I trial of a personalized peptide vaccine for patients positive for human leukocyte antigen-A24 with recurrent or progressive glioblastoma multiforme. J Clin Oncol 29: 337-44, 2011

14) Weller M, Butowski N, Tran D, et al.: ATIM-03. ACT IV. An international double-blind, phase 3 trial of rindopepimut in newly diagnosed, EGFRvIIIexpressing glioblastoma. Neuro Oncol 18 (suppl_6): vi17-vi18, 2016.

15) Yamanaka R, Itoh K: Peptide-based immunotherapeutic approaches to glioma: a review. Expert Opin Biol Ther 7: 645-649, 2007 
16) Hayes RL, Arbit E, Odaimi M, et al.: Adoptive cellular immunotherapy for the treatment of malignant gliomas. Crit Rev Oncol Hematol 39: 31-42, 2001

17) Ishikawa E, Takano S, Ohno $\mathrm{T}$, Tsuboi K: Adoptive cell transfer therapy for malignant gliomas. $A d v$ Exp Med Biol 746: 109-120, 2012

18) Lillehei $\mathrm{KO}$, Mitchell $\mathrm{DH}$, Johnson SD, McCleary EL, Kruse CA: Long-term follow-up of patients with recurrent malignant gliomas treated with adjuvant adoptive immunotherapy. Neurosurgery 28: 16-23, 1991

19) Yoshida S, Tanaka R, Takai N, Ono K: Local administration of autologous lymphokine-activated killer cells and recombinant interleukin 2 to patients with malignant brain tumors. Cancer Res 48: 5011-5016, 1988

20) Ishikawa E, Tsuboi K, Saijo K, et al.: Autologous natural killer cell therapy for human recurrent malignant glioma. Anticancer Res 24: 1861-1871, 2004

21) Tsuboi K, Saijo K, Ishikawa E, et al.: Effects of local injection of ex vivo expanded autologous tumor-specific T-lymphocytes in cases with recurrent malignant gliomas. Clin Cancer Res 9: 3294-3302, 2003

22) Dudley ME, Yang JC, Sherry R, et al.: Adoptive cell therapy for patients with metastatic melanoma: evaluation of intensive myeloablative chemoradiation preparative regimens. J Clin Oncol 26: 5233-5239, 2008

23) Grupp SA, Kalos M, Barrett D, et al.: Chimeric antigen receptor-modified $\mathrm{T}$ cells for acute lymphoid leukemia. N Engl J Med 368: 1509-1518, 2013

24) Ohno M, Natsume A, Ichiro Iwami K, et al.: Retrovirally engineered T-cell-based immunotherapy targeting type III variant epidermal growth factor receptor, a glioma-associated antigen. Cancer Sci 101: 2518-2524, 2010

25) Sengupta S, Mao G, Gokaslan ZS, Sampath P: Chimeric antigen receptors for treatment of glioblastoma. a practical review of challenges and ways to overcome them. Cancer Gene Ther Oct 21, 2016. [Epub ahead of print]

26) Mineharu Y, Castro MG, Lowenstein PR, Sakai N, Miyamoto S: Dendritic cell-based immunotherapy for glioma: multiple regimens and implications in clinical trials. Neurol Med Chir (Tokyo) 53: 741-754, 2013

27) Di Lorenzo G, Ferro M, Buonerba C: Sipuleucel-T (Provenge $\left.{ }^{\circledR}\right)$ for castration-resistant prostate cancer. BJU Int 110(2 Pt 2): E99-E104, 2012

28) Ohno T: Autologous formalin-fixed tumor vaccine. Curr Pharm Des 11: 1181-1188, 2005

29) Ishikawa E, Yamamoto T, Sakamoto N, et al.: Low peripheral lymphocyte count before focal radiotherapy plus concomitant temozolomide predicts severe lymphopenia during malignant glioma treatment. Neurol Med Chir (Tokyo) 50: 638-644, 2010
30) Sampson JH, Aldape KD, Archer GE, et al.: Greater chemotherapy-induced lymphopenia enhances tumor-specific immune responses that eliminate EGFRvIII-expressing tumor cells in patients with glioblastoma. Neuro-oncology 13: 324-333, 2011

31) Kim TG, Kim CH, Park JS, et al.: Immunological factors relating to the antitumor effect of temozolomide chemoimmunotherapy in a murine glioma model. Clin Vaccine Immunol 17: 143-153, 2010

32) Sanchez-Perez LA, Choi BD, Archer GE, et al.: Myeloablative temozolomide enhances CD8 ${ }^{+}$T-cell responses to vaccine and is required for efficacy against brain tumors in mice. PLoS One 8: e59082, 2013

33) Liu G, Black KL, Yu JS: Sensitization of malignant glioma to chemotherapy through dendritic cell vaccination. Expert Rev Vaccines 5: 233-247, 2006

34) Sakamoto N, Ishikawa E, Yamamoto T, et al.: Pathological changes after autologous formalin-fixed tumor vaccine therapy combined with temozolomide for glioblastoma - three case reports. Neurol Med Chir (Tokyo) 51: 319-325, 2011

35) Ishikawa E, Muragaki Y, Yamamoto T, et al.: Phase I/IIa trial of chemoradiotherapy plus autologous formalin-fixed tumor vaccine for newly diagnosed glioblastoma. Association between complete resection and delayed-type hypersensitivity response. J Clin Oncol 32 (suppl; abstr e13029), 2014

36) Aruga A, Takeshita N, Kotera Y, et al.: Long-term vaccination with multiple peptides derived from cancer-testis antigens can maintain a specific T-cell response and achieve disease stability in advanced biliary tract cancer. Clin Cancer Res 19: 2224-2231, 2013

37) Pardoll DM: The blockade of immune checkpoints in cancer immunotherapy. Nat Rev Cancer 12: 252-264, 2012

38) Zhang $X$, Zhu S, Li T, Liu YJ, Chen W, Chen J: Targeting immune checkpoints in malignant glioma. Oncotarget Oct 16, 2016

39) Taube JM, Klein A, Brahmer JR, et al.: Association of PD-1, PD-1 ligands, and other features of the tumor immune microenvironment with response to anti-PD-1 therapy. Clin Cancer Res 20: 5064-5074, 2014

40) Wang A, Wang HY, Liu Y, et al.: The prognostic value of PD-L1 expression for non-small cell lung cancer patients: a meta-analysis. Eur J Surg Oncol 41: 450-456, 2015

41) Nduom EK, Wei J, Yaghi NK, et al.: PD-L1 expression and prognostic impact in glioblastoma. Neurooncology 18: 195-205, 2016

42) Berghoff AS, Kiesel B, Widhalm G, et al.: Programmed death ligand 1 expression and tumor-infiltrating lymphocytes in glioblastoma. Neuro-oncology 17: 1064-75, 2015

43) Miyazaki T, Ishikawa E, Matsuda M, et al.: Assessment of PD-1 positive cells on initial and secondary resected tumor specimens of newly diagnosed glio- 
blastoma and its implications on patient outcome. J Neurooncol 2017. [Epub ahead of print]

44) Verbrugge I, Galli M, Smyth MJ, Johnstone RW, Haynes NM: Enhancing the antitumor effects of radiotherapy with combinations of immunostimulatory antibodies. Oncoimmunology 1: 1629-1631, 2012

45) Hamid O, Robert C, Daud A, et al.: Safety and tumor responses with lambrolizumab (anti-PD-1) in melanoma. $N$ Engl J Med 369: 134-144, 2013

46) Melero I, Grimaldi AM, Perez-Gracia JL, Ascierto PA: Clinical development of immunostimulatory monoclonal antibodies and opportunities for combination. Clin Cancer Res 19: 997-1008, 2013

47) Sampson JH, Vlahovic G, Desjardins A, et al.: Randomized phase IIb study of nivolumab (anti-PD-1; BMS-936558, ONO-4538) alone or in combination with ipilimumab versus bevacizumab in patients (pts) with recurrent glioblastoma (GBM). J Clin Oncol 32:5s (suppl; abstr TPS2101), 2014

48) https://clinicaltrials.gov/ct2/show/NCT02667587, Study of Temozolomide Plus Radiation Therapy With Nivolumab or Placebo, for Newly Diagnosed Patients With Glioblastoma. (CheckMate548), ClinicalTrials.gov (Accessed on 2016 Dec 26)

49) Sampson JH, Omuro A, Preusser M, et al.: A randomized, phase 3, open-label study of nivolumab versus temozolomide in combination with radiotherapy in adult patients with newly diagnosed, O-6-methylguanine DNA methyltransferase-unmethylated glioblastoma: CheckMate-498. Clin Oncol 34 (suppl; abstr TPS2079), 2016

50) Kalbasi A, June CH, Haas N, Vapiwala N: Radiation and immunotherapy. a synergistic combination. J Clin Invest 123: 2756-2763, 2013

51) Hodge JW, Guha C, Neefjes J, Gulley JL: Synergizing radiation therapy and immunotherapy for curing incurable cancers. Opportunities and challenges. Oncology (Williston Park, NY) 22: 1064-1070, 2008

52) Newcomb EW, Demaria S, Lukyanov Y, et al.: The combination of ionizing radiation and peripheral vaccination produces long-term survival of mice bearing established invasive GL261 gliomas. Clin Cancer Res 12: 4730-4737, 2006

53) Park SD, Kim CH, Kim CK, et al.: Cross-priming by temozolomide enhances antitumor immunity of dendritic cell vaccination in murine brain tumor model. Vaccine 25: 3485-3491, 2007

54) Huang Y, Goel S, Duda DG, Fukumura D, Jain RK: Vascular normalization as an emerging strategy to enhance cancer immunotherapy. Cancer Res 73: 2943-2948, 2013

55) Huang Y, Yuan J, Righi E, et al.: Vascular normalizing doses of antiangiogenic treatment reprogram the immunosuppressive tumor microenvironment and enhance immunotherapy. Proc Natl Acad Sci USA 109: 17561-17566, 2012

56) Sakamoto N, Uemae Y, Ishikawa E, et al.: Glioma immunotherapy with combined autologous tumor cell and endothelial cell vaccine in vivo. Neurol Med Chir (Tokyo) 52: 194-201, 2012

57) Tartour E, Pere H, Maillere B, et al.: Angiogenesis and immunity: a bidirectional link potentially relevant for the monitoring of antiangiogenic therapy and the development of novel therapeutic combination with immunotherapy. Cancer Metastasis Rev 30: 83-95, 2011

58) Avril T, Saikali S, Vauleon E, et al.: Distinct effects of human glioblastoma immunoregulatory molecules programmed cell death ligand-1 (PDL-1) and indoleamine 2,3-dioxygenase (IDO) on tumour-specific T cell functions. J Neuroimmunol 225: 22-33, 2010

59) Terawaki S, Chikuma S, Shibayama S, et al.: IFN- $\alpha$ directly promotes programmed cell death-1 transcription and limits the duration of $\mathrm{T}$ cell-mediated immunity. J Immunol 186: 2772-2779, 2011

60) Mandai M, Hamanishi J, Abiko K, Matsumura N, Baba T, Konishi I: Dual Faces of IFN- $\gamma$ in Cancer Progression. A Role of PD-L1 Induction in the Determination of Pro- and Antitumor Immunity. Clin Cancer Res 22: 2329-34, 2016

61) Ali OA, Lewin SA, Dranoff G, Mooney DJ: Vaccines Combined with Immune Checkpoint Antibodies Promote Cytotoxic T-cell Activity and Tumor Eradication. Cancer Immunol Res 4: 95-100, 2016.

62) Karyampudi L, Lamichhane P, Scheid AD, et al.: Accumulation of memory precursor CD8 T cells in regressing tumors following combination therapy with vaccine and anti-PD-1 antibody. Cancer Res 74: 2974-2985, 2014

63) Drake CG: Combination immunotherapy approaches. Ann Oncol 23 Suppl 8: viii41-6, 2012

64) Duraiswamy J, Freeman GJ, Coukos G: Dual blockade of PD-1 and CTLA-4 combined with tumor vaccine effectively restores T-cell rejection function in tumors-response. Cancer Res 74: 633-634, 2014

65) Hodi FS, Butler M, Oble DA, et al.: Immunologic and clinical effects of antibody blockade of cytotoxic $\mathrm{T}$ lymphocyte-associated antigen 4 in previously vaccinated cancer patients. Proc Natl Acad Sci USA 105: 3005-3010, 2008

66) Miyoshi T, Kataoka T, Asahi A, et al.: A transient increase and subsequent sharp decrease of chemorefractory liver-metastasized uterine cervical small cell carcinoma to autologous formalin-fixed tumor vaccine plus anti-PD-1 antibody. Clin Case Rep 4: 687-691, 2016

67) Zeng J, See AP, Phallen J, et al.: Anti-PD-1 blockade and stereotactic radiation produce long-term survival in mice with intracranial gliomas. Int J Radiat Oncol Biol Phys 86: 343-349, 2013

68) Antonios JP, Soto H, Everson RG, et al.: PD-1 blockade enhances the vaccination-induced immune response in glioma. JCI Insight 1: pii: e87059, 2016

69) Wainwright DA, Chang AL, Dey M, et al.: Durable therapeutic efficacy utilizing combinatorial blockade 
against IDO, CTLA-4, and PD-L1 in mice with brain tumors. Clin Cancer Res 20: 5290-5301, 2014

70) Bertrand A, Kostine M, Barnetche T, Truchetet ME, Schaeverbeke T: Immune related adverse events associated with anti-CTLA-4 antibodies: systematic review and meta-analysis. BMC Med 13: 211, 2015

71) Weber JS, Postow M, Lao CD, Schadendorf D: Management of adverse events following treatment with anti-programmed death-1 agents. Oncologist 21: 1230-1240, 2016
72) Larkin J, Chiarion-Sileni V, Gonzalez R, et al.: Combined nivolumab and ipilimumab or monotherapy in untreated melanoma. $N$ Engl J Med 373: 23-34, 2015

Address reprint requests to: Eiichi Ishikawa, $\mathrm{MD}, \mathrm{PhD}$, Department of Neurosurgery, Faculty of Medicine, University of Tsukuba, 1-1-1 Tennodai, Tsukuba, Ibaraki 305-8575, Japan.

e-mail: e-ishikawa@md.tsukuba.ac.jp 